\title{
Generalized Detector as Spectrum Sensor in Cognitive Radio Networks
}

\author{
Modar SHBAT ${ }^{1}$, Vyacheslav TUZLUKOV ${ }^{2}$ \\ School of Electronics Engineering, Kyungpook National University, 1370 Sankyuk-dong, Bug-gu, Daegu, South Korea \\ modboss80@gmail.com, tuzlukov@ee.knu.ac.kr
}

\begin{abstract}
The implementation of the generalized detector $(G D)$ in cognitive radio (CR) systems allows us to improve the spectrum sensing performance in comparison with employment of the conventional detectors. We analyze the spectrum sensing performance for the uncorrelated and spatially correlated receive antenna array elements. Additionally, we consider a practical case when the noise power at the output of GD linear systems (the preliminary and additional filters) is differed by value. The choice of the optimal GD threshold based on the minimum total error rate criterion is also discussed. Simulation results demonstrate superiority of GD implementation in CR system as spectrum sensor in comparison with the energy detector (ED), weighted ED (WED), maximum-minimum eigenvalue (MME) detector, and generalized likelihood ratio test (GLRT) detector.
\end{abstract}

\section{Keywords}

Cognitive radio (CR), spectrum sensing, generalized detector (GD), energy detector (ED), probability of false alarm

\section{Introduction}

Cognitive radio (CR) system is considered as a component of wireless network. CR systems promise us to get the efficient spectrum utilization and other advantages, for instance, the cell throughput enhancement [1].The CR user equipment (UE) can utilize an idle spectrum of the primary user (PU) network (see Fig. 1). Thus, the spectrum sensing is needed to define the idle spectrum. The secondary user (SU) network compiles the frequency holes that are found by spectrum sensing in the licensed band and opportunistically exploits these frequency holes for CR use. The spectrum sensing should assure that the SU does not generate any interference to the PU network. Most of spectrum sensing approaches are based on implementation of the energy detector (ED) [2] and [3], matched filter [4] and [5], and cyclostationary detector [6] and [7]. Complete knowledge about the PU signal is required in the case of the matched filter. The cyclostationary detector can exploit the PU signal features caused by periodicity. The ED does not need any a priori information about the PU signal and can be used for spectrum sensing even in the case when the sensing time is less than $1 \mathrm{~ms}$ [1]. On the other hand, the $\mathrm{ED}$ is sensitive with respect to the noise power uncertainty and spatial correlation between the antenna array elements that can cause a serious deterioration in the ED performance at the low signal-to-noise ratio $(S N R)$.

Many attempts to improve the ED spectrum sensing performance are made at the present time, for example, the ED with dynamic threshold [8], ED with two-step threshold [9], weighted ED (WED) [10], cooperative spectrum sensing, i.e. sharing the sensing results between some secondary users [11], [12], and so on. Other spectrum sensing techniques are also investigated, for example, the generalized likelihood ratio test (GLRT) detector [13], maximumminimum eigenvalue (MME) detector [14], and spectrum sensing based on known PU signal pattern using the preamble and midamble [1], the radio identification procedure [15], and the two phase algorithms for spectrum sensing and power/rate control of a SU [16]. Useful information about different spectrum sensing algorithms can be found in [1]. The ED is commonly used as a spectrum sensor owing to low computational cost, simplicity in implementation, and no a priori information about the PU signal is needed. Unfortunately, the ED detection performance degradation is evident under the spatially correlated antenna array elements [10].

In this paper, we address the coarse spectrum sensing problem in the CR systems employing the generalized detector (GD) constructed based on the generalized approach

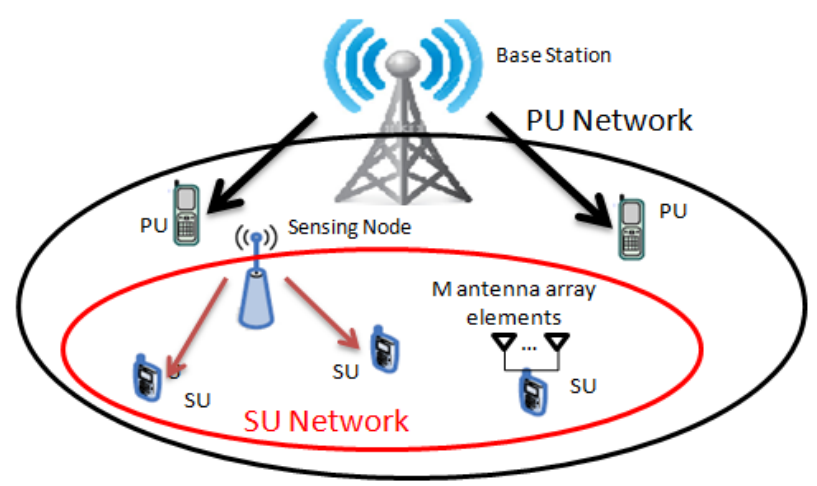

Fig. 1. The $\mathrm{CR}$ system and the $\mathrm{SU}$ with $M$ antenna array elements. 
to signal processing (GASP) in noise [17] under the uncorrelated (no spatial correlation) and spatially correlated antenna array elements. The GD formulates a test statistics based on definition of the jointly sufficient statistics of the likelihood ratio mean and variance [17-19]. The GD performance in wireless communication and radar sensor systems is discussed in [19-27]. The first attempt to study the GD implementation as a spectrum sensor in CR networks is discussed in [28].

In the present paper, we compare the GD, ED, WED, GLRT detector, and MME detector by spectrum sensing performance. Comparing with [28], we investigate the case of inequality between the noise power at the outputs of GD input linear systems, namely, the preliminary filter (PF) and additional filter (AF). This case is very close to practice. Additionally, the GD optimal detection threshold is defined based on the minimum probability of error criterion under two cases, namely, the noise powers or variances at the output of GD input linear systems (the PF and $\mathrm{AF}$ ) are the same and different by value. Effect of the coefficient of spatial correlation between the receive antenna array elements on the GD spectrum sensing performance is also investigated.

The rest of the paper is divided into the following sections. The model of spatial correlation between the antenna array elements is discussed in Sec. 2. The GD main structure and the test statistics are presented in Sec. 3. Definition of the GD threshold and probability of false alarm under the independent (uncorrelated) and spatially correlated antenna array elements are delivered in Sec. 4. The optimal detection threshold and probability of false alarm for ED are briefly discussed in Sec. 5. The GD optimal threshold selection is defined in Sec. 6. Simulation results are discussed in Sec. 7. The conclusions are delivered in Sec. 8 .

\section{CR System Model}

The spectrum sensing is performed by the SU in the CR network system with $M$ receive antenna array elements Each $i$-th antenna array element receives $N$ samples during the sensing time. The sensing problem can be described by the binary hypotheses:

$$
z_{i}[k]= \begin{cases}w_{i}[k], & i=1, \ldots, M ; k=0, \ldots, N-1 \Rightarrow H_{0} ; \\ h_{i}[k] s[k]+w_{i}[k], & i=1, \ldots, M ; k=0, \ldots, N-1 \Rightarrow H_{1},\end{cases}
$$

where $z_{i}[k]$ is the discrete-time received signal at the SU input; $s[k]$ is the discrete-time modulated transmitted signal with the same probability of transmission for all symbols (the PU signal); $h_{i}[k]$ is the channel coefficient obeying the circularly symmetric complex Gaussian distribution with zero mean and variance equal to $\sigma_{h}{ }^{2}$, i.e. $h_{i}[k] \sim C N\left(0, \sigma_{h}{ }^{2}\right)$; and $w_{i}[k]$ is the discrete-time circularly symmetric complex Gaussian noise with zero mean and variance $\sigma_{w}{ }^{2}$, i.e. $w_{i}[k] \sim C N\left(0, \sigma_{w}{ }^{2}\right)$. The channel parameters are not varied during the sensing time (coarse or fast sensing) and the channel coefficients $h_{i}[k]$ are spatially correlated between each other but independent over the time. The modulated signal $s[k]$, channel coefficients $h_{i}[k]$, and AWGN $w_{i}[k]$ are independent between each other. The same channel model is widely used in [29-31].

Owing to its simplicity, the exponential matrix model is widely used to describe the spatial correlation between the adjacent antenna array elements [32]. The components of the $M \times M$ antenna array element correlation matrix $\mathbf{C}$ can be presented in the following form:

$$
\mathbf{C}_{i j}=\left\{\rho^{i-j}\right\}, \quad i \leq j, \quad i, j=1, \ldots, M,
$$

where $\rho$ is the coefficient of spatial correlation between the adjacent antenna array elements $(0 \leq \rho \leq 1$, the real values). Applying the results presented in [32], the coefficient of spatial correlation $\rho$ can be given as

$$
\rho=\exp \left\{-23 \Lambda^{2}(d / \lambda)^{2}\right\}
$$

where $\Lambda$ is the angular spread, an important propagation parameter defining a distribution of multipath power of radio waves coming in at the receiver input from a number of azimuthal directions with respect to the horizon; $\lambda$ is the wavelength; and $d$ is the distance between two adjacent antenna array elements (antenna array element spacing). The correlation matrix of antenna array elements $\mathbf{C}$ given by (2) is the symmetric Toeplitz matrix [29].

The $M N \times 1$ signal vector $\mathbf{Z}$ received by $M$ receive antenna array elements consists of all the observed signals during the sensing time and can be defined using the following form:

$$
\mathbf{Z}=\left[z_{1}[0], \ldots, z_{M}[0], \ldots, z_{1}[N-1], \ldots, z_{M}[N-1]\right]^{T}
$$

where $T$ denotes a transpose. The received signal vector $\mathbf{Z}$ has a complex Gaussian distribution with the covariance matrices $\mathbf{C o v}_{\mathbf{0}}$ and $\mathbf{C o v}_{\mathbf{1}}$ under the hypotheses $H_{0}$ and $H_{1}$ respectively. If $z_{i}[k]=w_{i}[k]$, the received signals $z_{i}[k]$ are independent. Under the hypothesis $H_{1}$, if $z_{i}[k]=h_{i}[k] s[k]$ $+w_{i}[k]$, the received signals are spatially correlated. The covariance matrices $\mathbf{C o v}_{\mathbf{0}}$ and $\mathbf{C o v}_{\mathbf{1}}$ are determined in [28].

\section{GD Flowchart and Decision Statistics}

\subsection{Main Functioning Principles of GD and Test Statistics}

The GD is a linear combination of the correlation detector, which is optimal in the Neyman-Pearson (NP) criterion sense when a priori information about the PU signal parameters is known, and the energy detector, which is optimal in the NP criterion sense if the PU signal parameters have a random character [17], [18]. As was discussed in detail in [18, Chapter 7, p. 685-692], the main function of the GD energy detector (GD ED) is to detect the PU 


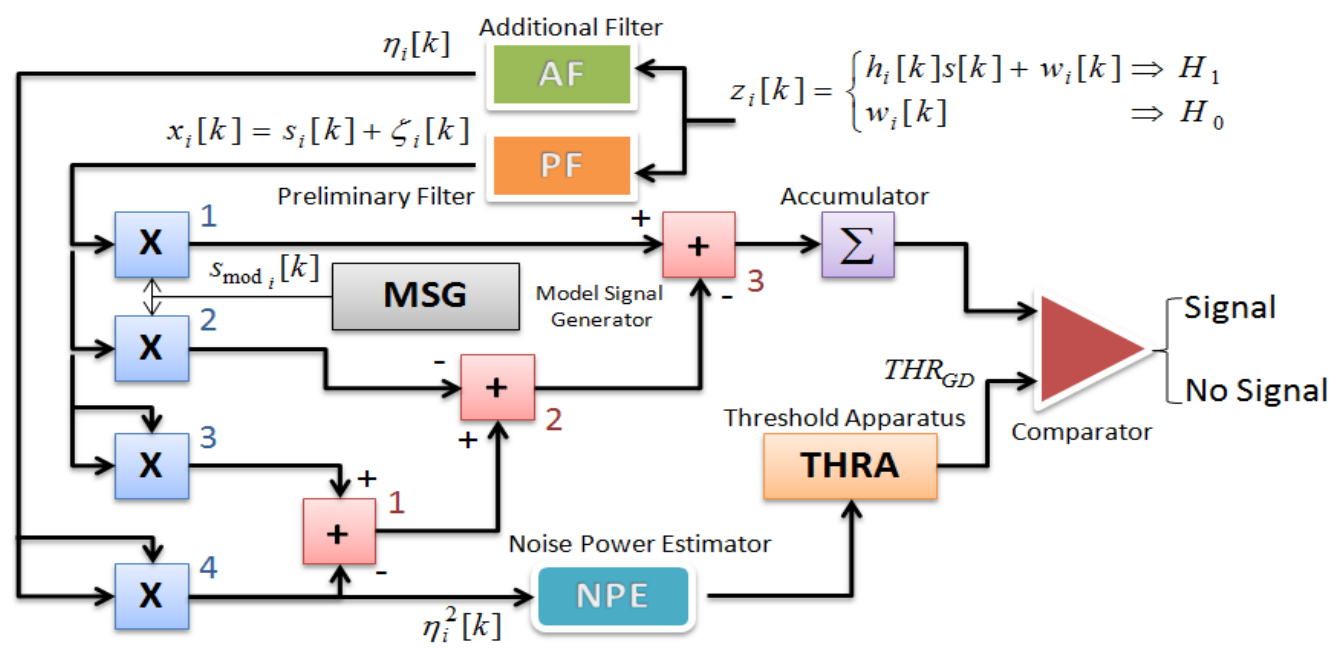

Fig. 2. The GD structure.

signal. The main function of the GD correlation detector is to define the detected PU signal parameters and make a decision: the detected signal is the expected and desired PU signal or not. A great difference between the GD ED and conventional ED employed by the classical signal detection theory is a presence of the additional filter (AF) at the GD front-end, i.e. the GD input linear system.

The specific feature of GASP is introduction of additional noise source that does not carry any information about the signal with the purpose to improve a qualitative signal detection performance. This additional noise can be considered as the reference noise without any information about the signal to be detected. The jointly sufficient statistics of the mean and variance of the likelihood ratio is obtained in the case of GASP implementation, while the classical and modern signal processing theories can deliver only a sufficient statistics of the mean or variance of the likelihood ratio (no the jointly sufficient statistics of the mean and variance of the likelihood function). Thus, the implementation of GASP allows us to obtain more information about the input process or received information signal. Owing to this fact, an implementation of the receivers constructed based on GASP basis allows us to improve the spectrum sensing performance of CR networks in comparison with employment of other conventional receivers.

The GD flowchart is presented in Fig. 2. As we can see from Fig. 2, the GD consists of three channels:

- the GD correlation detector channel- the PF, multipliers 1 and 2, model signal generator (MSG);

- the GD ED channel - the PF, AF, multiplier 3 and 4, summator 1 ;

- the compensation channel - the summators 2 and 3 and the accumulator.

For description of the GD flowchart we consider the discrete-time processes without loss of any generality. The main purpose of the GD compensation channel is to cancel the GD correlation detector channel noise component $s_{\text {modi }_{i}}[k] \xi_{i}[k]$ and the GD ED channel random component $s_{i}[k] \xi_{i}[k]$ between each other based on the same nature of the noise $\xi_{i}[k]$. Evidently, this cancelation is possible only based on the same nature of the noise $\xi_{i}[k]$ satisfying the condition of equality between the signal model $s_{\bmod _{i}}[k]$ and incoming signal $s_{i}[k]$ over the whole range of parameters, i.e.

$$
s_{\bmod _{i}}[k]=s_{i}[k]
$$

that is the GD main functioning condition. To satisfy (5), we are able to define the incoming signal parameters. Naturally, in practice, the signal parameters are random. The condition (5) is important for complete compensation in the statistical sense between the noise component of the GD correlation detector channel $2 s_{\text {mod }_{i}}[k] \xi_{i}[k]$, caused by interaction between the model signal $s_{\bmod _{i}}[k]$ and noise $\xi_{i}[k]$, and the random component of the GD ED channel $2 s_{i}[k] \xi_{i}[k]$, caused by interaction between the signal $s_{i}[k]$ and noise $\xi_{i}[k][17]$ and [18, Chapter 3]. The complete matching between the model signal $s_{\text {mod }_{i}}[k]$ and the incoming signal $s_{i}[k]$, especially by amplitude, is a very hard problem in practice and only in the ideal case the complete matching is possible. How the GR detection performance can be deteriorated under mismatching between the model signal $s_{\text {mod }_{i}}[k]$ and the incoming signal $s_{i}[k]$ is discussed in [33]. How we can satisfy (5) in practice is discussed in detail in [17] and [18, Chapter 7, p. 631-695] when there is no a priori information about the signal $s_{i}[k]$.

The practical implementation of the GD decision statistics requires an estimation of the noise variance $\sigma_{w}{ }^{2}$ using the reference noise $\eta_{i}[k]$ at the AF output. The AF is the reference noise source. The PF bandwidth is matched with the bandwidth of the information signal $s_{i}[k]$ to be detected. The threshold apparatus (THRA) device defines the GD threshold.

The PF and AF can be considered as the bandpass filters with the impulse responses $h_{P F}[m]$ and $h_{A F}[m]$, respectively. For simplicity of analysis, we assume that these 
filters have the same amplitude-frequency characteristics or impulse responses by shape. Moreover, the AF central frequency is detuned with respect to the PF central frequency on such a value that the information signal cannot pass through the AF. If a value of detuning between the AF and $\mathrm{PF}$ central frequencies is more than 4 or $5 \Delta f_{s}$, where $\Delta f_{s}$ is the signal bandwidth, the processes at the AF and $\mathrm{PF}$ outputs can be considered as the uncorrelated and independent processes and, in practice, under this condition, the coefficient of correlation between the PF and AF output processes is not more than 0.05 that was confirmed experimentally in [34], [35].

If the noise $w[k]$ at the PF and AF inputs is Gaussian, the noise at the PF and AF outputs is Gaussian, too, because the PF and AF are the linear systems that do not change the statistical parameters of the input process. A detailed discussion of the AF and PF can be found in [36]. The noise at the PF and AF outputs can be presented in the following form:

$$
\left\{\begin{array}{l}
w_{P F}[k]=\xi[k]=\sum_{m=-\infty}^{\infty} h_{P F}[m] w[k-m] \\
w_{A F}[k]=\eta[k]=\sum_{m=-\infty}^{\infty} h_{A F}[m] w[k-m]
\end{array}\right.
$$

Under the hypothesis $H_{1}$ the signal at the PF output can be defined as $x_{i}[k]=s_{i}[k]+\xi_{i}[k]$ (see Fig. 2), where $\xi_{i}[k]$ is the observed noise at the PF output and

$$
s_{i}[k]=h_{i}[k] \times s[k] ;
$$

$h_{i}[k]$ is the channel coefficient. Under the hypothesis $H_{0}$ and for all $i$ and $k$, the process $x_{i}[k]=\xi_{i}[k]$ at the PF output is subjected to the complex Gaussian distribution and can be considered as the independent and identically distributed (i.i.d.) process. The process at the AF output is the reference noise $\eta_{i}[k]$ with the same statistical parameters as the noise $\xi_{i}[k]$ in the ideal case. In practice, the statistical parameters of the noise $\xi_{i}[k]$ and $\eta_{i}[k]$ can be differed.

The decision statistics at the GD output presented in [17], [18] is extended to the case of antenna array employment and can be presented in the following form:

$$
\begin{aligned}
& T_{G D}(\mathbf{X})= \sum_{k=0}^{N-1} \sum_{i=1}^{M} 2 x_{i}[k] s_{\bmod _{i}}[k]-\sum_{k=0}^{N-1} \sum_{i=1}^{M} x_{i}^{2}[k] \\
&+\sum_{k=0}^{N-1} \sum_{i=1}^{M} \eta_{i}^{2}[k]>_{H_{0}}^{H_{1}} T H R_{G D} ; \\
& \mathbf{X}=\left[x_{1}[0], \ldots, x_{M}[0], \ldots, x_{1}[N-1], \ldots, x_{M}[N-1]\right]^{T}
\end{aligned}
$$

is the vector of PF output random process; $T H R_{G D}$ is the detection threshold of GD. We can rewrite (8) using the vector form:

$$
\begin{gathered}
T_{G D}(\mathbf{X})=2 \mathbf{S}_{\bmod } \mathbf{X}-\mathbf{X}^{2}+\boldsymbol{\eta}^{2} \gtrless_{H_{0}}^{H_{1}} T H R_{G D} ; \\
\mathbf{X}=[\mathbf{x}(0), \ldots, \mathbf{x}(N-1)]
\end{gathered}
$$

is the $M \times 1$ vector of the random process at the PF output with elements defined as

$$
\begin{gathered}
\mathbf{x}[k]=\left[x_{1}[k], \ldots, x_{M}[k]\right]^{T} ; \\
\mathbf{S}_{\text {mod }}=\left[\mathbf{s}_{\text {mod }}(0), \ldots, \mathbf{s}_{\text {mod }}(N-1)\right]
\end{gathered}
$$

is the $M \times 1$ vector of the process at the MSG output with the elements defined as

$$
\begin{aligned}
\mathbf{s}_{\bmod }[k] & =\left[s_{\bmod _{1}}[k], \ldots, s_{\bmod _{M}}[k]\right]^{T} ; \\
\boldsymbol{\eta} & =[\boldsymbol{\eta}(0), \ldots, \boldsymbol{\eta}(N-1)]
\end{aligned}
$$

is the $M \times 1$ vector of the random process at the AF output with the elements defined as

$$
\boldsymbol{\eta}[k]=\left[\eta_{1}[k], \ldots, \eta_{M}[k]\right]^{T}
$$

and $T H R_{G D}$ is the GD detection threshold.

According to GASP and GD structure shown in Fig. 2, the GD test statistics takes the following form under the hypotheses $H_{1}$ and $H_{0}$, respectively:

$$
T_{G D}(\mathbf{X})= \begin{cases}\sum_{k=0}^{N-1} \sum_{i=1}^{M} s_{i}^{2}[k]+\sum_{k=0}^{N-1} \sum_{i=1}^{M} \eta_{i}^{2}[k]-\sum_{k=0}^{N-1} \sum_{i=1}^{M} \xi_{i}^{2}[k] & \Rightarrow H_{1}, \\ \sum_{k=0}^{N-1} \sum_{i=1}^{M} \eta_{i}^{2}[k]-\sum_{k=0}^{N-1} \sum_{i=1}^{M} \xi_{i}^{2}[k] & \Rightarrow H_{0} .\end{cases}
$$

The term $\sum_{k=0}^{N-1} \sum_{i=1}^{M} s_{i}^{2}[k]=E_{s}$ is the average signal energy and the term $\sum_{k=0}^{N-1} \sum_{i=1}^{M} \eta_{i}^{2}[k]-\sum_{k=0}^{N-1} \sum_{i=1}^{M} \xi_{i}^{2}[k]$ presents the background noise at the GD output that is a difference between the noise power at the PF and AF outputs.

\subsection{Moment Generation Function of the GD Partial Test Statistics $T_{G R}\left(\mathbf{X}_{k}\right)$}

To analyze the GD performance as a spectrum sensor, there is a need to define the moment generating function (MGF) of the partial test statistics $T_{G R}\left(\mathbf{X}_{k}\right)$

$$
T_{G R}\left(\mathbf{X}_{k}\right)=\sum_{i=1}^{M} s_{i}^{2}[k]+\sum_{i=1}^{M} \eta_{i}^{2}[k]-\sum_{i=1}^{M} \xi_{i}^{2}[k]
$$

under the main GD functioning condition (5) and the hypothesis $H_{1}$.We say that the random variable $x$ has a chisquare distribution with $v$ degree of freedom if its probability density function (pdf) is determined as

$$
p(x)=c x^{0.5 v-1} \exp (-0.5 x)
$$

where $c$ is a constant given by [37]

$$
c=\frac{1}{2^{0.5 v} \Gamma(0.5 v)},
$$

$\Gamma(\cdot)$ is the gamma function. The MGF general form for the chi-square distributed random variable $x$ is given by 


$$
\begin{aligned}
M_{x}(l) & =E[\exp (l x)]=\int_{-\infty}^{\infty} \exp (l x) p(x) d x \\
& =c \int_{0}^{\infty} \exp (l x) x^{0.5 v-1} \exp (-0.5 x) d x .
\end{aligned}
$$

At $v=1$, the constant can be presented given by

$$
c=\frac{1}{2^{0.5} \Gamma(0.5 v)}=\frac{1}{\sqrt{2 \pi}} .
$$

Assume that $z_{1 i}[k]=\xi_{i}^{2}[k]$ and $z_{2 i}[k]=\eta_{i}^{2}[k]$. The pdfs for the random variables $z_{1 i}$ and $z_{2 i}$ are defined by the chi-square $\chi^{2}$ distribution law with one degree of freedom [18]:

$$
\begin{gathered}
p\left(z_{1 i}\right)=\frac{1}{\sqrt{2 \pi z_{1 i}} \sigma_{w}} \exp \left\{-\frac{z_{1 i}}{2 \sigma_{w}^{2}}\right\}, z_{1 i}>0, \\
p\left(z_{2 i}\right)=\frac{1}{\sqrt{2 \pi z_{2 i}} \sigma_{w}} \exp \left\{-\frac{z_{2 i}}{2 \sigma_{w}^{2}}\right\}, z_{2 i}>0 .
\end{gathered}
$$

Introduce a new random variable $z_{i}=z_{1 i}-z_{2 i}$. MGF of the random variable $z_{i}$ is given using the following formula:

$M_{z_{i}}(l)=E\left[\exp \left(l z_{1 i}\right)\right]=E\left\{\exp \left[l\left(z_{1 i}-z_{2 i}\right)\right]\right\}$

$=E\left[\exp \left(l z_{1 i}\right) \exp \left(-l z_{2 i}\right)\right]=E\left[\exp \left(l z_{1 i}\right)\right] E\left[\exp \left(-l z_{2 i}\right)\right]$

$=M_{z_{1 i}}(l) M_{z_{2 i}}(-l)$.

MGF of the random variable $z_{1}$ is defined in the following form:

$$
\begin{aligned}
& M_{z_{1 i}}(l)=\frac{1}{\sqrt{2 \pi} \sigma_{w}} \int_{0}^{\infty} \exp \left(l z_{1 i}\right) z_{1 i}^{-0.5} \exp \left\{-\frac{z_{1 i}}{2 \sigma_{w}^{2}}\right\} d z_{1 i} \\
& =\frac{1}{\sqrt{2 \pi} \sigma_{w}} \int_{0}^{\infty} z_{1 i}^{-0.5} \exp \left\{-\left(\frac{1}{2 \sigma_{w}^{2}}-l\right) z_{1 i}\right\} d z_{1 i} .
\end{aligned}
$$

Introducing the variable $g_{i}=\left(0.5 \sigma_{w}^{-2}-l\right) z_{1 i}$, we obtain

$$
\begin{aligned}
& M_{z_{1 i}}(l)=\frac{2 \sigma_{w}^{2}}{\sqrt{2 \pi} \sigma_{w}} \\
& \times \int_{0}^{\infty}\left[2 \sigma_{w}^{2}\left(1-2 \sigma_{w}^{2} l\right)^{-1}\right]^{-\frac{1}{2}} g_{i}^{-0.5} \exp \left(-g_{i}\right)\left(1-2 \sigma_{w}^{2} l\right)^{-1} d g_{i} \\
& =\frac{1}{\sqrt{2 \pi \sigma_{w}}}\left[2 \sigma_{w}^{2}\left(1-2 \sigma_{w}^{2} l\right)^{-1}\right]^{-\frac{1}{2}} \int_{0}^{\infty} g_{i}^{-0.5} \exp \left(-g_{i}\right) d g_{i} \\
& =\left[\pi\left(1-2 \sigma_{w}^{2} l\right)\right]^{-\frac{1}{2}} \int_{0}^{\infty} g_{i}^{-0.5} \exp \left(-g_{i}\right) d g_{i} .
\end{aligned}
$$

Based on definition of the gamma function [38]

$$
\Gamma(x)=\int_{0}^{\infty} l^{x-1} \exp (-l) d l
$$

we obtain that

$$
\int_{0}^{\infty} g_{i}^{-0.5} \exp \left(-g_{i}\right) d g_{i}=\Gamma(0.5)=\sqrt{\pi} .
$$

Finally, the MGF of the random variable $z_{1 i}$ is defined as

$$
M_{z_{1 i}}(l)=\frac{\sqrt{\pi}}{\sqrt{\pi\left(1-2 \sigma_{w}^{2}\right)}}=\frac{1}{\sqrt{1-2 \sigma_{w}^{2} l}} .
$$

The mean and variance of the random variables $z_{1_{i}}$ can be determined in the following form:

$E\left[z_{1 i}\right]=\left.\frac{\partial M_{z_{1 i}}(l)}{\partial l}\right|_{l=0}=\sigma_{w}^{2}$,

$\operatorname{Var}\left[z_{1 i}\right]=E\left[z_{1 i}^{2}\right]-E\left[z_{1 i}\right]^{2}=\left.\frac{\partial^{2} M_{z_{1 i}}(l)}{\partial^{2} l}\right|_{l=0}-E\left[z_{1 i}\right]^{2}$

$=3 \sigma_{w}^{4}-\sigma_{w}^{4}=2 \sigma_{w}^{4}$.

By analogous way, we can find that the MGF of the random variable $z_{2 i}$ takes the following form:

$$
M_{z_{2 i}}(-l)=\frac{1}{\sqrt{1+2 \sigma_{w}^{2} l}} .
$$

Since $\left\{s_{i}[k]\right\}_{i=1}^{M}$ are spatially correlated for $i$-th antenna array elements, according to [36] the MGF of $\sum_{i=1}^{M} s_{i}^{2}[k]$ is defined as

$$
M_{\sum_{i=1}^{M} s_{i}^{2}[k]}(l)=\prod_{i=1}^{M}\left(1-E_{s} \sigma_{h}^{2} \beta_{i} l\right)^{-1}
$$

where $\beta_{i}$ is the eigenvalue of the $i$-th spatial channel of the correlation matrix $\mathbf{C}$ given by (2). Based on (18), (21), and (22) the MGF of the GD partial decision statistics $T_{G D}\left(\mathbf{X}_{k}\right)$ is determined in the following form:

$$
\begin{aligned}
& M_{T_{G D}\left(\mathbf{x}_{k}\right)}(l)=\prod_{i=1}^{M}\left[1-E_{s} \sigma_{h}^{2} \beta_{i} l\right]^{-1} \prod_{i=1}^{M} M_{z_{1 i}}(l) \prod_{i=1}^{M} M_{z_{z_{i}}}(-l) \\
& =\prod_{i=1}^{M}\left[1-E_{s} \sigma_{h}^{2} \beta_{i} l\right]^{-1} \prod_{i=1}^{M}\left(1-2 \sigma_{w}^{2} l\right)^{-0.5} \prod_{i=1}^{M}\left(1+2 \sigma_{w}^{2} l\right)^{-0.5} \\
& =\prod_{i=1}^{M}\left[1-E_{s} \sigma_{h}^{2} \beta_{i} l\right]^{-1}\left(1-2 \sigma_{w}^{2} l\right)^{-0.5 M}\left(1+2 \sigma_{w}^{2} l\right)^{-0.5 M} \\
& =\left(1-4 \sigma_{w}^{4} l^{2}\right)^{-0.5 M} \prod_{i=1}^{M}\left[1-E_{s} \sigma_{h}^{2} \beta_{i} l\right]^{-1} .
\end{aligned}
$$

\section{GD Spectrum Sensing}

\subsection{Spatially Correlated Antenna Array Elements}

The GD defines the total received PU signal energy 
during the sensing time and compares it with the detection threshold $T H R_{G D}$ to make a decision about the PU signal presence or absence at the SU input. Under the hypothesis $H_{0}$ according to the initial conditions, the mean and variance of the test statistics $T_{G D}(\mathbf{X})$ at the GD output are given by [18, Chapter 3]:

$$
\left\{\begin{array}{l}
m_{H_{0}}^{G D}=E\left[T_{G D}(\mathbf{X}) \mid H_{0}\right]=0, \\
\operatorname{Var}_{H_{0}}^{G D}=\operatorname{Var}\left[T_{G D}(\mathbf{X}) \mid H_{0}\right]=4 N M \sigma_{w}^{4},
\end{array}\right.
$$

where $N$ is the number of samples and $M$ is the number of antenna array elements.

Applying the central limit theorem, the GD test statistics under the hypothesis $H_{0}$ can be approximated by the normal Gaussian distribution as $N \rightarrow \infty$ :

$$
H_{0} \Rightarrow T_{G D}(\mathbf{X}) \sim N\left(0,4 N M \sigma_{w}^{4}\right) .
$$

Based on (35), the mean and the variance of the test statistics $T_{G D}(\mathbf{X})$ under the hypothesis $H_{1}$ take the following form, respectively:

$$
\begin{gathered}
m_{H_{1}}^{G D}=E\left[T_{G D}\left(\mathbf{X}_{k}\right) \mid H_{1}\right]=N M E_{s} \sigma_{h}^{2} \\
\operatorname{Var}_{H_{1}}^{G D}=\operatorname{Var}\left[T_{G D}\left(\mathbf{X}_{k}\right) \mid H_{1}\right]=N\left[\sum_{i=1}^{M} E_{s}^{2} \sigma_{h}^{4} \beta_{i}^{2}+4 M \sigma_{w}^{4}\right]
\end{gathered}
$$

As $N \rightarrow \infty$, applying the central limit theorem, the pdf of the GD test statistics $T_{G D}(\mathbf{X})$ under the hypothesis $H_{1}$ takes the following form:

$$
H_{1} \Rightarrow T_{G D}(\mathbf{X}) \sim N\left\{N M E_{s} \sigma_{h}^{2}, N\left[\sum_{i=1}^{M} E_{s}^{2} \sigma_{h}^{4} \beta_{i}^{2}+4 M \sigma_{w}^{4}\right]\right\}
$$

Thus, as $N \rightarrow \infty$, based on the central limit theorem, we can apply the approximation by the normal Gaussian pdf for the GD test statistics to define the probability of false alarm $P_{F A}^{G D}$ and the probability of detection $P_{D}^{G D}$. In [18] and [36], the probability of false alarm $P_{F A}^{G D}$ and the probability of miss $P_{\text {miss }}^{G D}$ are defined in the following form:

$$
\begin{aligned}
& P_{F A}^{G D}=P\left(T_{G D}(\mathbf{X}) \geq T H R_{G D} \mid H_{0}\right)=1-\Phi\left(\frac{T H R_{G D}-m_{H_{0}}^{G D}}{\sqrt{\operatorname{Var}_{H_{0}}^{G D}}}\right), \\
& P_{m i s s}^{G D}=P\left(T_{G D}(\mathbf{X})<T H R_{G D} \mid H_{1}\right)=\Phi\left(\frac{T H R_{G D}-m_{H_{1}}^{G D}}{\sqrt{\operatorname{Var}_{H_{1}}^{G D}}}\right),
\end{aligned}
$$

where [38]

$$
\Phi(x)=\frac{1}{2}+\frac{1}{2} \operatorname{erf}\left(\frac{x}{\sqrt{2}}\right)
$$

is the integral of probability and

$$
\operatorname{erf}(x)=\int_{0}^{x} \exp \left(-t^{2}\right) d t
$$

is the error function; $m_{H_{0}}^{G D}$ is the mean of the test statistics $T_{G D}(\mathbf{X})$ under the hypothesis $H_{0} ; \operatorname{Var}_{H_{0}}^{G D}$ is the variance of the test statistics $T_{G D}(\mathbf{X})$ under the hypothesis $H_{0} ; m_{H_{1}}^{G D}$ is the mean of the test statistics $T_{G D}(\mathbf{X})$ under the hypothesis $H_{1}$; and $\operatorname{Var}_{H_{1}}^{G D}$ is the variance of the test statistics $T_{G D}(\mathbf{X})$ under the hypothesis $H_{1}$. Alternatively, the probability of false alarm $P_{F A}^{G D}$ and the probability of detection $P_{D}^{G D}$ can be defined as:

$$
\begin{aligned}
& P_{F A}^{G D}=P\left(T_{G D}(\mathbf{X}) \geq T H R_{G D} \mid H_{0}\right)=Q\left(\frac{T H R_{G D}-m_{H_{0}}^{G D}}{\sqrt{\operatorname{Var}_{H_{0}}^{G D}}}\right), \\
& P_{D}^{G D}=P\left(T_{G D}(\mathbf{X})>T H R_{G D} \mid H_{1}\right)=Q\left(\frac{T H R_{G D}-m_{H_{1}}^{G D}}{\sqrt{\operatorname{Var}_{H_{1}}^{G D}}}\right),
\end{aligned}
$$

where

$$
P_{D}^{G D}=1-P_{m i s s}^{G D}
$$

and $Q(x)$ is the Gaussian $Q$-function given by

$$
Q(x)=\frac{1}{\sqrt{2 \pi}} \int_{x}^{\infty} \exp \left(-0.5 t^{2}\right) d t=\frac{1}{2}-\frac{1}{2} \operatorname{erf}\left(\frac{x}{\sqrt{2}}\right) .
$$

To avoid a generation of interference for PU, it is important to define a lower bound for the probability of detection $P_{D}$. In this case, the sensing performance is evaluated according to the probability of false alarm $P_{F A}$ while maintaining the predetermined lower bound of the probability of detection $P_{D}$. For this purpose, there is a need to define the GD threshold $T H R_{G D}$ as a function of the probability of detection $P_{D}$ applying the required lower bound, i.e. $P_{D}^{G D} \geq \alpha$, where $\alpha$ is the constraint.

In practice, the noise variance can be estimated by the noise power estimator (NPE, see Fig. 2) using the reference noise at the AF output. Based on (46), the GD threshold $T H R_{G D}$ can be determined at the probability of detection constraint in the following form:

$$
T H R_{G D}=m_{H_{1}}^{G D}+\sqrt{\operatorname{Var}_{H_{1}}^{G D}} Q^{-1}(\alpha)
$$

As follows from (38)-(40), the GD threshold $T H R_{G D}$ given by (49) can be rewritten in the following form:

$$
T H R_{G D}=N M E_{s} \sigma_{h}^{2}+Q^{-1}(\alpha) \sqrt{N\left\{\sum_{i=1}^{M} E_{s}^{2} \sigma_{h}^{4} \beta_{i}^{2}+4 M \sigma_{w}^{4}\right\}}
$$

Defining the $S N R$ at the SU input as

$$
\gamma=E_{s} \sigma_{h}^{2} / \sigma_{w}^{2}
$$

we can rewrite (50) as

$$
T H R_{G D}=N M \gamma \sigma_{w}^{2}+Q^{-1}(\alpha) \sqrt{N\left\{\sum_{i=1}^{M} \gamma^{2} \sigma_{w}^{4} \beta_{i}^{2}+4 M \sigma_{w}^{4}\right\}}
$$


Based on (45), taking into consideration the probability of detection constraint, we can find that the probability of false alarm $P_{F A}^{G D}$ under the spatially correlated antenna array elements takes the following form:

$$
\begin{aligned}
P_{F A}^{G D_{c o r}}= & Q\left\{\frac { 1 } { \sqrt { 4 N M \sigma _ { w } ^ { 4 } } } \left[N M E_{s} \sigma_{h}^{2}+Q^{-1}(\alpha)\right.\right. \\
& \left.\left.\times \sqrt{N\left\{\sum_{i=1}^{M} E_{s}^{2} \sigma_{h}^{4} \beta_{i}^{2}+4 M \sigma_{w}^{4}\right\}}\right]\right\} .
\end{aligned}
$$

Using (51), we can represent the probability of false alarm $P_{F A}^{G D_{c o r}}$ in the following form:

$$
P_{F A}^{G D_{\text {cor }}}=Q\left\{\frac{1}{2}\left[\gamma \sqrt{N M}+\frac{1}{Q(\alpha)} \sqrt{\frac{1}{M}\left(\sum_{i=1}^{M} \gamma^{2} \beta_{i}^{2}+4 M\right)}\right]\right\} .
$$

\subsection{Uncorrelated Antenna Array Elements}

In general, the antenna array elements can be considered as independent or uncorrelated $(\rho=0)$ when the ratio $d / \lambda$ is high by value and the angular spread $\Lambda$ tends to approach $\pi$. Under this condition, the correlation matrix $\mathbf{C}$ given by (2) becomes the $M \times M$ identity matrix, i.e. $\mathbf{I}_{M \times M}$. In this case, taking into consideration the probability of detection constraint, the probability of false alarm for independent or uncorrelated antenna array elements $P_{F A}^{u n c o r}$ can be defined based on the probability of false alarm for the spatially correlated antenna array elements $P_{F A}^{c o r}$ as given by [29] and [30]:

$$
P_{F A}^{u n c o r}=\lim _{\rho \rightarrow 0} P_{F A}^{c o r} .
$$

Based on (54) and (55), taking into consideration the probability of detection constraint, the probability of false alarm $P_{F A}^{G D}$ under independent or uncorrelated antenna array elements can be determined as

$$
P_{F A}^{G D_{\text {uncor }}}=\lim _{\rho \rightarrow 0} P_{F A}^{G D_{\text {cor }}}=Q\left\{\frac{1}{2}\left[Q^{-1}(\alpha) \sqrt{\left(\gamma^{2}+4\right)}+\gamma \sqrt{N M}\right]\right\} .
$$

For any values of $N, M$, and $\gamma$ we can see that:

$$
\begin{aligned}
& Q^{-1}\left(P_{F A}^{G D_{\text {uncor }}}\right)-Q^{-1}\left(P_{F A}^{G D_{c o r}}\right) \\
& \quad=\frac{1}{2} Q^{-1}(\alpha)\left[\sqrt{\left(\gamma^{2}+4\right)}-\sqrt{\frac{1}{M}\left(\sum_{i=1}^{M} \gamma^{2} \beta_{i}^{2}+4 M\right)}\right] \geq 0,
\end{aligned}
$$

since $Q(x)$ is the monotonically decreasing function of $x$ and $Q^{-1}(\alpha)$ is the negative value. Thus, (57) is the lower bound of the probability of false alarm $P_{F A}^{G D}$ for GD.

\subsection{Unequal Power at the PF and AF Outputs}

Before, we made assumption that the power or variance $\sigma_{\xi}^{2}$ of the noise $\xi_{i}[k]$ at the PF output is equal to the power or variance $\sigma_{\eta}^{2}$ of the reference noise $\eta_{i}[k]$ at the AF output, i.e., $\sigma_{\xi}{ }^{2}=\sigma_{\eta}{ }^{2}=\sigma_{w}{ }^{2}$. Based on this assumption, we could define the noise power estimation using only the noise sample $\eta_{i}[k]$ at the AF output with the purpose to define the variance of the background noise $\sum_{k=0}^{N-1} \sum_{i=1}^{M} \eta_{i}^{2}[k]-\sum_{k=0}^{N-1} \sum_{i=1}^{M} \xi_{i}^{2}[k]$ at the GD output given by (see (36))

$$
\operatorname{Var}\left[\sum_{k=0}^{N-1} \sum_{i=1}^{M} \eta_{i}^{2}[k]-\sum_{K=0}^{N-1} \sum_{i=1}^{M} \xi_{i}^{2}[k]\right]=4 N M \sigma_{w}^{4} .
$$

Naturally, in practice (see subsection 3.1), the equality between the variances of the noise $\xi_{i}[k]$ and $\eta_{i}[k]$ is impossible, i.e. $\sigma_{\xi}{ }^{2} \neq \sigma_{\eta}{ }^{2}$, owing to many reasons, for example, the instability of $\mathrm{PF}$ and $\mathrm{AF}$ parameters, nonuniform environment, nonhomogeneous noise, etc. By this reason, there is a need to consider the condition closed to practice when $\sigma_{\xi}^{2} \neq \sigma_{\eta}{ }^{2}$. Assume that

$$
\left\{\begin{array}{l}
\sigma_{\xi}^{2}=\sigma_{w}^{2} \\
\sigma_{\eta}^{2}=\mu \sigma_{\xi}^{2}=\mu \sigma_{w}^{2}
\end{array}\right.
$$

where $\mu$ is a factor of proportionality. Thus, the variance of the GD background noise can be presented using the following form:

$$
\operatorname{Var}\left[\sum_{k=0}^{N-1} \sum_{i=1}^{M} \eta_{i}^{2}[k]-\sum_{K=0}^{N-1} \sum_{i=1}^{M} \xi_{i}^{2}[k]\right]=2 N M \sigma_{w}^{4}\left(1+\mu^{2}\right) .
$$

The mean $m_{H_{0}}^{G D}$ and variance $\operatorname{Var}_{H_{0}}^{G D}$ of the test statistics $T_{G D}(\mathbf{X})$ under the hypothesis $H_{0}$ are determined as:

$$
\left\{\begin{array}{l}
m_{H_{0}}^{G D}=E\left[T_{G D}(\mathbf{X}) H_{0}\right]=0, \\
\operatorname{Var}_{H_{0}}^{G D}=\operatorname{Var}\left[T_{G D}(\mathbf{X}) H_{0}\right]=2 N M \sigma_{w}^{4}\left(1+\mu^{2}\right) .
\end{array}\right.
$$

Under the hypothesis $H_{1}$, the mean $m_{H_{1}}^{G D}$ and variance $\operatorname{Var}_{H_{1}}^{G D}$ of GD test statistics $T_{G D}(\mathbf{X})$ take the following form:

$$
\left\{\begin{array}{l}
m_{H_{1}}^{G D}=E\left[T_{G D}(\mathbf{X}) H_{1}\right]=N M E_{s} \sigma_{h}^{2}, \\
\operatorname{Var}_{H_{1}}^{G D}=\operatorname{Var}\left[T_{G D}(\mathbf{X}) H_{1}\right]=N M\left[E_{s}^{2} \sigma_{h}^{4}+2 \sigma_{w}^{4}\left(1+\mu^{2}\right)\right] .
\end{array}\right.
$$

Based on (62), we can define the GD threshold in the following form:

$$
\begin{aligned}
T H R_{G D} & =m_{H_{1}}^{G D}+\sqrt{\operatorname{Var}_{H_{1}}^{G D}} Q^{-1}(\alpha) \\
= & \sqrt{N M} \sigma_{w}^{2}\left\{\gamma \sqrt{N M}+Q^{-1}(\alpha) \sqrt{\gamma^{2}+2\left(1+\mu^{2}\right)}\right\} .
\end{aligned}
$$

The general form of the GD probability of false alarm is determined as 


$$
P_{F A}^{G D_{\text {uncor }}}=Q\left\{\frac{\gamma \sqrt{N M}+Q^{-1}(\alpha) \sqrt{\gamma^{2}+2\left(1+\mu^{2}\right)}}{\sqrt{2\left(1+\mu^{2}\right)}}\right\} .
$$

Note if $\mu=1$, (64) becomes (56).

\section{ED as a Spectrum Sensor}

Under the initial conditions discussed in Sec. 2, the energy of the received PU signal is combined with the equal gain at different antenna array elements. The test statistics at the ED output can be defined as

$$
T_{E D}(\mathbf{Z})=\sum_{k=0}^{N-1} \sum_{i=1}^{M}\left|z_{i}[k]\right|^{2} .
$$

According to [29], applying the central limit theorem the ED test statistics under the hypotheses $H_{0}$ and $H_{1}$ obeys the following distributions:

$$
\left\{\begin{array}{l}
T_{E D}^{H_{0}}(\mathbf{Z}) \sim N\left(N M \sigma_{w}^{2}, N M \sigma_{w}^{4}\right), \\
T_{E D}^{H_{1}}(\mathbf{Z}) \sim N\left\{N M\left(E_{s} \sigma_{h}^{2}+\sigma_{w}^{2}\right), N \sum_{i=1}^{M}\left(E_{s} \sigma_{h}^{2} \beta_{i}+\sigma_{w}^{2}\right)^{2}\right\}
\end{array}\right.
$$

Under spatially correlated antenna array elements, the ED probability of false alarm $P_{F A}^{E D}$ can be written as [31]:

$$
P_{F A}^{E D_{c o r}}=Q\left[Q^{-1}(\alpha) \sqrt{\frac{1}{M} \sum_{i=1}^{M}\left(\gamma \beta_{i}+1\right)^{2}}+\gamma \sqrt{N M}\right] .
$$

For the uncorrelated antenna array elements case, the ED probability of false alarm $P_{F A}^{E D}$ takes the form [29]:

$$
P_{F A}^{E D_{\text {uncor }}}=\lim _{\rho \rightarrow 0} P_{F A}^{E D_{\text {cor }}}=Q\left[Q^{-1}(\alpha)(\gamma+1)+\gamma \sqrt{N M}\right] .
$$

In the case of correlated antenna array elements, the implementation of the WED proposed in [10] allows us to improve the detection performance in comparison with the ED and other detectors constructed based on eigenvalues of the correlation matrix $\mathbf{C}$ such as the MME and GLRT detectors. If the noise variance $\sigma_{w}{ }^{2}$ is known, applying the Neyman-Pearson theorem [3], the likelihood ratio test is defined as

$$
L(\mathbf{Z})=\frac{p\left(\mathbf{Z} \mid H_{1}, \sigma_{w}^{2}\right)}{p\left(\mathbf{Z} \mid H_{0}, \sigma_{w}^{2}\right)},
$$

where $p\left(\mathbf{Z} \mid H_{1}, \sigma_{w}^{2}\right)$ and $p\left(\mathbf{Z} \mid H_{0}, \sigma_{w}^{2}\right)$ are the likelihood functions under the hypothesis $H_{1}$ and $H_{0}$, respectively. WED decision statistic is determined in the following form [10]:

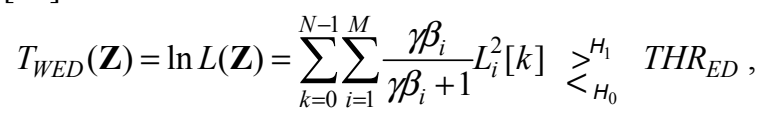

where $T H R_{E D}$ is the ED threshold; $L_{i}[k]$ is the linear transformation of observed data $z_{i}[k]$ given by $\mathbf{L}[k]=\mathbf{V}^{H} \mathbf{z}[k]$ with the $\mathbf{v}_{i}$ eigenvector of the correlation matrix $\mathbf{C}$ given by (2). The transformed signal component $L_{i}[k]$ corresponding to the large eigenvalue $\beta_{i}$ is weighted more heavily [10]. When the antenna array elements are uncorrelated, all the eigenvalues $\beta_{i}$ will be the same and the WED may be considered as the ED.

\section{Definition of Optimal GD Threshold}

\subsection{The case $\sigma_{\xi}^{2}=\sigma_{\eta}^{2}$}

The detection threshold is a parameter used to define the probability of false alarm $P_{F A}$ and the probability of detection $P_{D}$ or the probability of miss $P_{\text {miss }}$. It is always preferable to achieve the high probability of detection $P_{D}$ and the low probability of false alarm $P_{F A}$. Sometimes, in practice, it is impossible to define the optimal threshold satisfying these requirements. This problem can be considered as the optimization problem [40] similar to the optimal sensing time selection problem [41].

The optimal detection threshold can be derived based on the minimal probability of error $P_{e r}$. The probability of error $P_{e r}$ can be presented in the following form:

$$
P_{e r}=p_{0} P_{F A}+p_{1} P_{m i s s},
$$

where $p_{0}$ and $p_{1}=1-p_{0}$ are the a priori probabilities of the PU signal absence and presence, respectively. For simplicity of analysis, we assume that these a priori probabilities are known and equal between each other $p_{0}=p_{1}=0.5$. In the GD case, it is possible to minimize the probability of error $P_{e r}$ by appropriate choice of the GD detection threshold $T H R_{G D}$. Thus, the optimal GD detection threshold $T H R_{G D}^{o p}$ can be defined as

$$
T H R_{G D}^{o p}=\arg \min _{T H R_{G D}} P_{e r}\left(T H R_{G D}\right) .
$$

As follows from Appendix (see (96)), the optimal GD threshold is defined in a very simple form:

$$
T H R_{G D}^{o p}=2 N M \sigma_{w}^{2}=\sigma_{\eta}^{2}
$$

Thus, the normalized optimal GD threshold

$$
T H R_{G D_{\sigma_{\xi}^{2}=\sigma_{\eta}^{2}}^{o p}}^{o} \frac{T H R_{G D_{\sigma_{\xi}^{2}=\sigma_{\eta}^{2}}^{o p}}^{N M}}{N M}=2 \sigma_{w}^{2}
$$

is only a function of the noise variance at the GD input. The optimal ED threshold is defined in [42] as

$$
T H R_{E D}^{o p}=\sigma_{w}^{2} \text {. }
$$




\subsection{The case $\sigma_{\xi}^{2} \neq \sigma_{\eta}^{2}$}

The GD optimal threshold $T H R_{G D_{\sigma_{\xi}^{2} \neq \sigma_{\eta}^{2}}^{o p}}$ if the noise power at the PF and AF outputs are differed by the factor of proportionality $\mu$ is given by (see Appendix, (95)):

$$
T H R_{G D_{\sigma_{\xi}^{2} \neq \sigma_{\eta}^{2}}^{o p}}=N M \sigma_{w}^{2} \sqrt{2\left(1+\mu^{2}\right)}
$$

The normalized optimal GD threshold is determined in the following form:

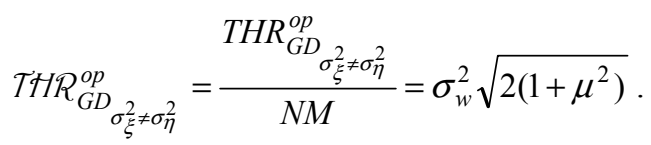

In this analysis we consider the additive white Gaussian noise (AWGN) channel. The same analysis steps can be applied to other kind of fading channels such as the Nakagami- $m$ fading channel and Rayleigh fading channel.

\section{Simulation Results}

Comparison between the GD spectrum sensing performance under the spatially correlated antenna array elements with other detectors, namely, ED, WED, MME detector, and GLRT detector is carried out using MATLAB following the standard simulation environment and parameters (IEEE 802.22). We assume that the GD input noise power or variance $\sigma_{w}{ }^{2}$ is known. We use the same main simulation parameters as in [29], where the angular spread $\Lambda=0.5^{\circ}$ in the case of spatially correlated antenna array elements with $d=\lambda / 8$ and $\Lambda=180^{\circ}$ for independent or uncorrelated antenna array elements with $d=\lambda / 2$, where $d$ is the distance between two adjacent antenna array elements (antenna array element spacing) and $\lambda$ is the wavelength; the channel parameter $\sigma_{h}^{2}=1$; and the probability of detection constraint $\alpha=0.99$.

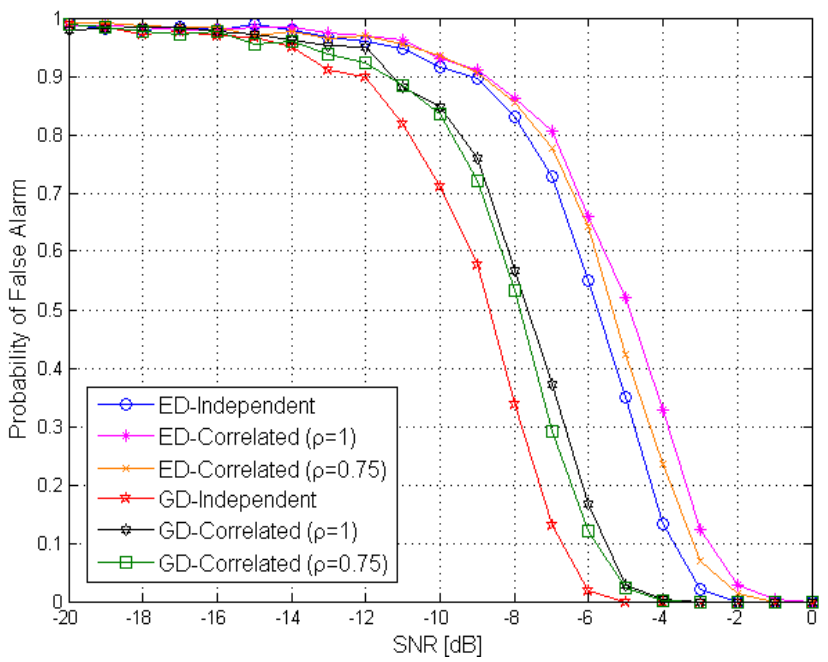

Fig. 3. Comparison of spectrum sensing performance between the ED and GD.
The ED and GD performances are compared in Fig. 3 under the independent (uncorrelated) and spatially correlated antenna array elements with the coefficient of correlation $\rho$ equal to 1 and $0.75 ; M=6$ and $N=20$. The GD demonstrates the better spectrum sensing performance in comparison with the ED for all cases. For example, under the spatially correlated antenna array elements at the coefficient of correlation $\rho=1$ and the probability of false alarm $P_{F A}=0.5$, the $S N R$ gain in favor of GD is approximately equal to $2.5 \mathrm{~dB}$ in comparison with the ED. When the antenna array elements are independent (uncorrelated), the $S N R$ gain is about $3 \mathrm{~dB}$ in favor of GD comparing with the ED at the probability of false alarm $P_{F A}=0.5$. In general, as we can see from Fig. 3 , the probability of false alarm $P_{F A}$ for the spatially correlated antenna array elements both for the ED and GD is high in comparison with the case when the antenna array elements are independent (uncorrelated) and the probability of false alarm $P_{F A}$ increases with increase in the coefficient of correlation $\rho$.

The curves characterizing the probability of miss as a function of the probability of false alarm, the complementary receiver operating characteristic (ROC) for the GD and ED are presented in Fig. 4 if the antenna array elements are both uncorrelated and spatially correlated at $M=6, N=20, S N R=-5 \mathrm{~dB}$ (Fig. 4a), and $S N R=-10 \mathrm{~dB}$ (Fig. 4b). The GD demonstrates superiority in sensing performance in comparison with the ED. For example, in the case of uncorrelated antenna array elements at the probability of false alarm $P_{P A}=10^{-2}$ and $S N R=-5 \mathrm{~dB}$ (Fig. 4a), in the case of ED, the probability of miss $P_{m i s s}^{E D}$ is approximately equal to 0.18 , while in the case of GD, the probability of miss $P_{m i s s}^{G D}$ is equal to 0.05 . Under the spatially correlated antenna array elements with the coefficient of correlation $\rho$ equal to $1, S N R=-5 \mathrm{~dB}$ (Fig. $4 \mathrm{a}$ ), and the probability of false alarm $P_{F A}$ equal to $10^{-2}$, the probability of miss $P_{m i s s}^{E D}$ is equal to 0.28 , and the probability of miss $P_{m i s s}^{G D}$ is approximately equal to 0.11 .

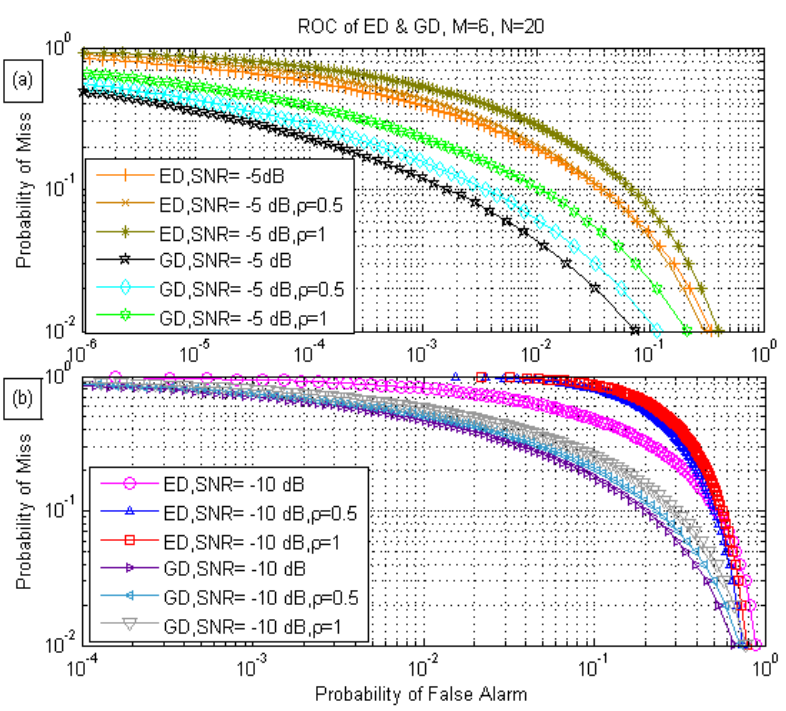

Fig. 4. Complementary ROC for ED and GD: a) $S N R=-5 \mathrm{~dB}$ and b) $S N R=-10 \mathrm{~dB}$. 
The composite hypothesis testing with the GLRT is used when there is an uncertainty with respect to one or more parameters in the likelihood functions, for instance, when the noise variance $\sigma_{w}{ }^{2}$ or/and the received PU signal covariance matrix $\mathbf{R}_{z}$ are unknown. In this case, we should obtain the maximum likelihood estimate (MLE) of the unknown parameter under the hypotheses $H_{0}$ and $H_{1}$ [13]:

$$
\left\{\begin{array}{l}
\hat{\sigma}_{w}^{2}=\arg \max _{\sigma_{w}^{2}} p\left(\mathbf{Z} \mid H_{0}, \sigma_{w}^{2}\right), \\
\hat{\mathbf{R}}_{z}=\arg \max _{\mathbf{R}_{z}} p\left(\mathbf{Z} \mid H_{1}, \mathbf{R}_{z}\right) .
\end{array}\right.
$$

Thus, the GLRT decision statistics can be presented in the following form:

$$
L_{G L R T}(\mathbf{Z})=\frac{p\left(\mathbf{Z} \mid \hat{\mathbf{R}}_{z}, H_{1}\right)}{p\left(\mathbf{Z} \mid \hat{\sigma}_{w}^{2}, H_{0}\right)} .
$$

More information about the GLRT detector and its spectrum sensing performance can be found in [10] and [13]. In the case of MME detector [13], [14], the maximum $\beta_{\max }$ and minimum $\beta_{\min }$ eigenvalues of the PU signal covariance matrix $\mathbf{R}_{y}$ are used to define the related MME decision statistics as follows [14]:

$$
T_{M M E}(\mathbf{X})=\frac{\beta_{\max }}{\beta_{\min }} .
$$

Thus, if there is no PU signal $\beta_{\max } / \beta_{\min }=1$, otherwise, we obtain $\beta_{\max } / \beta_{\text {min }}>1$.

Figure 5 demonstrates a comparison of the spectrum sensing performance for the WED, GLRT detector, MME detector, and GD under the spatially correlated antenna array elements with the coefficient of correlation $\rho$ equal to 1 at $M=6$ and $N=20$. As we can see from Fig. 5 , the spectrum sensing performances of the WED and GD are close to each other at $S N R<-14 \mathrm{~dB}$ with slightly vantage to the GD. At $S N R>-14 \mathrm{~dB}$, the GD performance is evidently better in comparison with WED one. If the probability of false alarm $P_{F A}$ is equal to 0.5 , the GD achieves the

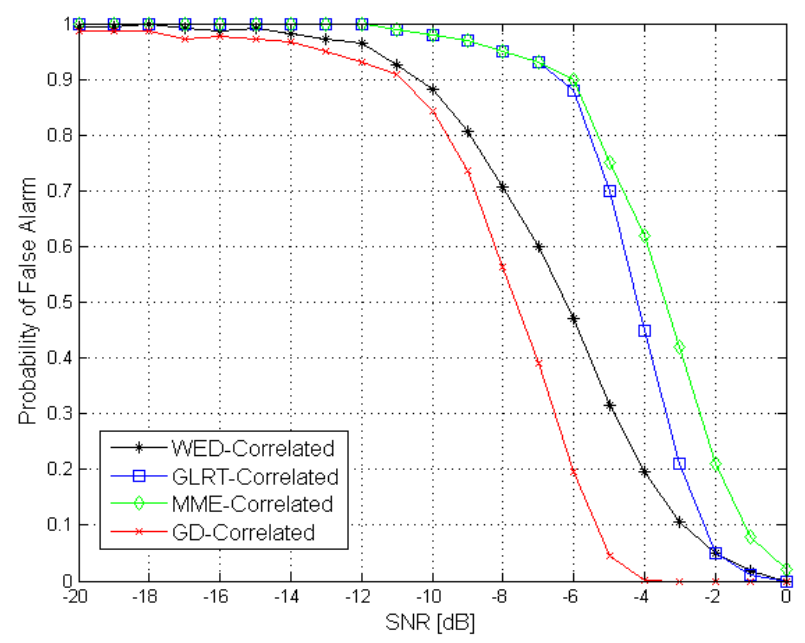

Fig. 5. Comparison of spectrum sensing performance between the WED, GLRT detector, MME detector, and GD.
$S N R$ gain equal approximately to $1.5 \mathrm{~dB}, 3.2 \mathrm{~dB}$, and $4.0 \mathrm{~dB}$ in comparison with the WED, GLRT detector, and MME detector, respectively.

The ED and GD spectrum sensing performances (the complementary ROC curves) obtained at $S N R=-10 \mathrm{~dB}$, $M=6, N=20$ are presented in Fig. 6 for the case $\sigma_{\xi}^{2} \neq \sigma_{\eta}^{2}$ i.e. $\sigma_{\eta}{ }^{2}=\mu \sigma_{\xi}^{2}=\mu \sigma_{w}{ }^{2}(59)$ at $\mu=0.5 ; 0.9 ; 1.5$. As we can see from Fig. 6 , the case $\mu \neq 1$, i.e. $\sigma_{\xi}^{2} \neq \sigma_{\eta}^{2}$, affects negatively on the GD performance and we can observe performance degradation in comparison with the case $\mu=1, \sigma_{\xi}{ }^{2}=\sigma_{\eta}{ }^{2}$. The GD stability to variations of $\mu$ is observed at low SNR values. This observation becomes clear from (64) at $\gamma<<1$ when the approximation $\gamma^{2}+2\left(1+\mu^{2}\right) \approx 2\left(1+\mu^{2}\right)$ leading us to reduction of the $\mu$ effect on the GD performance can be applied.

Figure 7 represents the probability of false alarm $P_{F A}$ as a function of $S N R$ for the ED and GD. The curves are shown at $M=6$ and $N=20$. In the GD case, the spectrum sensing performance is presented both for the case of $\sigma_{\xi}^{2}=\sigma_{\eta}^{2}$ and the case of $\sigma_{\xi}^{2} \neq \sigma_{\eta}^{2}$ at $\mu=0.5 ; 0.9 ; 1.5$. Naturally, we observe the best GD performance at $\mu=1$ or

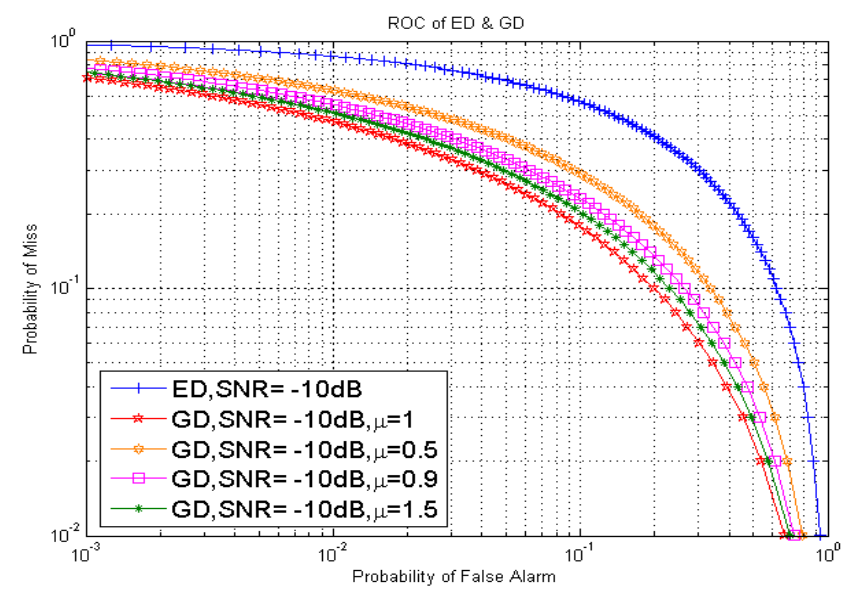

Fig. 6. Complementary ROC of ED and GD for the case $\sigma_{\xi}^{2} \neq \sigma_{\eta}^{2}$.

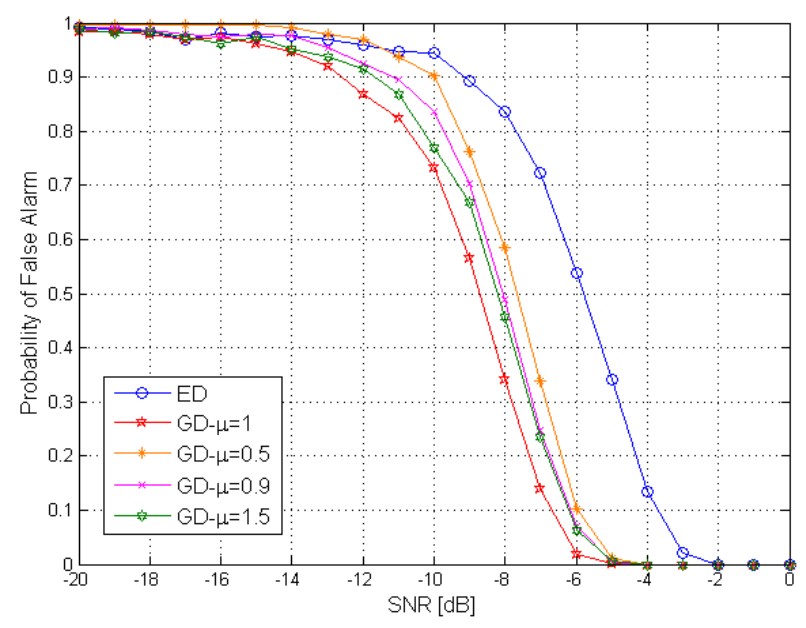

Fig. 7. ED and GD spectrum sensing performance when $\sigma_{\xi}^{2}=\sigma_{\eta}^{2}$ and $\sigma_{\xi}^{2} \neq \sigma_{\eta}^{2}$. 
$\sigma_{\xi}{ }^{2}=\sigma_{\eta}{ }^{2}$. As we can see from Fig. 7, a superiority of GD employment in CR networks in comparison with $\mathrm{ED}$ is evident both for the ideal case, $\sigma_{\xi}^{2}=\sigma_{\eta}^{2}$ and for the practical situation, $\sigma_{\xi}{ }^{2} \neq \sigma_{\eta}{ }^{2}$. Thus, the GD performance presented in Fig. 6 is confirmed by the simulation results presented in Fig. 7.

Figure 8 demonstrates the probabilities of false alarm $P_{F A}^{E D}$ and $P_{F A}^{G D}$ as a function of the number $M$ of spatially correlated antenna array elements. The number $M$ is varied from 1 to 10 . The coefficient $\rho$ of spatial correlation between the antenna array elements takes a value within the limits of the interval $[0.1,1.0]$.

The ED and GD spectrum sensing performances are presented at $S N R=-5 \mathrm{~dB} ;-10 \mathrm{~dB} ;-15 \mathrm{~dB}$. As we can see from Fig. 8, the probability of false alarm $P_{F A}$ increases monotonically with increase in the coefficient of correlation $\rho$. The large number of antenna array elements $M$ allows us to reduce the negative effect of the coefficient of spatial correlation $\rho$ on the probability of false alarm $P_{F A}$ for both detectors. The ED and GD performances have similar character under the spatial correlation between the antenna array elements, but it is evident that the GD has the better performance in comparison with the ED.

Comparison of the probability of error $P_{e r}$ for the ED and GD is presented in Fig. 9 in the case of independent or uncorrelated antenna array elements. The probability of error $P_{e r}$ as a function of the normalized optimal detection threshold, the normalization factor is taken as $N M$, is evaluated for the GD and ED at $S N R=-5 \mathrm{~dB} ;-10 \mathrm{~dB}$. The GD achieves the low probability of error $P_{e r}$ equal to 0.38 at the normalized threshold equal to $0.43 ; S N R=-5 \mathrm{~dB}$. The minimal ED probability of error $P_{e r}=0.63$ is achieved at the normalized threshold equal to $0.105 ; S N R=-5 \mathrm{~dB}$. Based on the observed GD and ED performances we conclude that the GD can achieve the lower probability of error $P_{e r}$ in comparison with the ED at the same $S N R$. Increasing in the $S N R$ allows us to minimize the probability

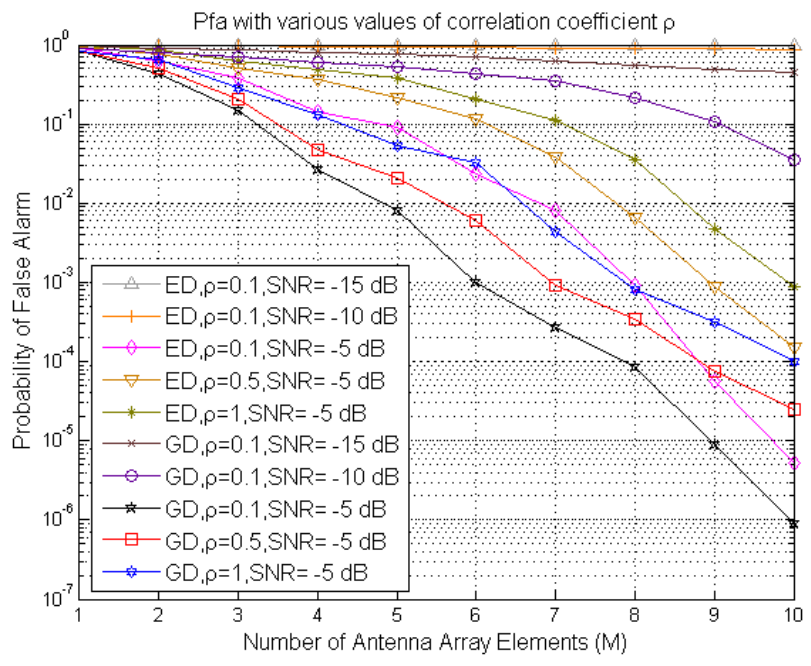

Fig. 8. The probabilities of false alarm $P_{F A}^{E D}$ and $P_{F A}^{G D}$ versus the number of antenna array elements $M$. of error $P_{e r}$ for both detectors. For example, an increase in the $S N R$ from $-10 \mathrm{~dB}$ to $-5 \mathrm{~dB}$ reduces the probability of error $P_{e r}$ from 0.88 to 0.63 in the ED case and from 0.74 to 0.38 in the GD case.

The probability of error $P_{e r}$ for GD as a function of the normalized optimal threshold given by (77) (the normalization factor is equal to $N M$ ) is shown in Fig. 10 for the case $\sigma_{\xi}^{2} \neq \sigma_{\eta}^{2}, \mu=0.5 ; 0.9 ; 1.5$ (the case in practice). As it follows from Fig. 10, we can observe that the GD performance degradation is caused by the negative effect of inequality between the variances, i.e. $\sigma_{\xi}^{2} \neq \sigma_{\eta}^{2}$, in comparison with the ideal case $\sigma_{\xi}^{2}=\sigma_{\eta}{ }^{2}$. In spite of this fact, the GD presents the better performance comparing with the ED one and demonstrates robustness against the noise variance inequality problem, $\sigma_{\xi}^{2} \neq \sigma_{\eta}^{2}$.

The noise variance inequality problem can be considered as a kind of limitation for the GD employment in CR networks. Another kind of limitation can be the interfering
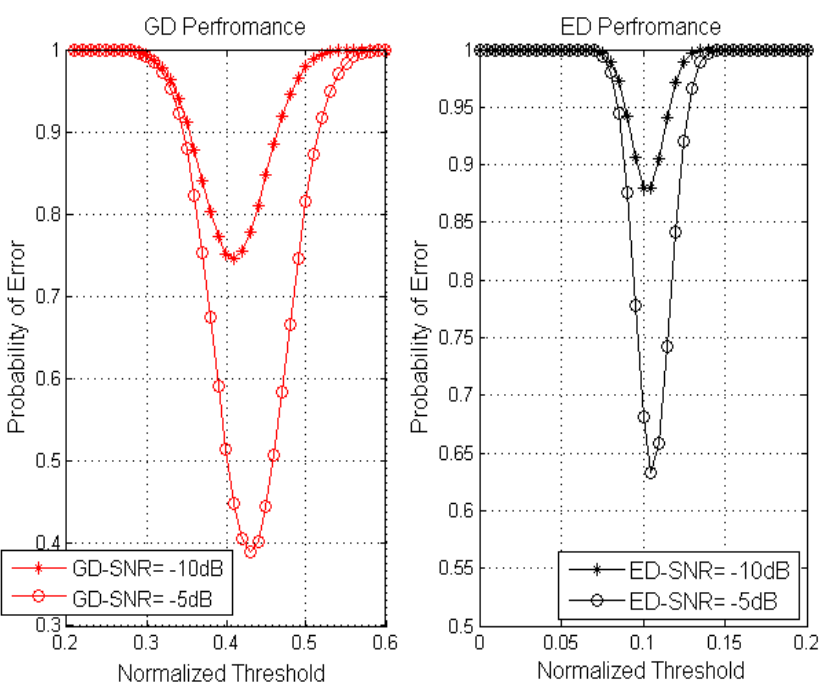

Fig. 9. The probability of error $P_{e r}$ for ED and GD as a function of the normalized threshold.

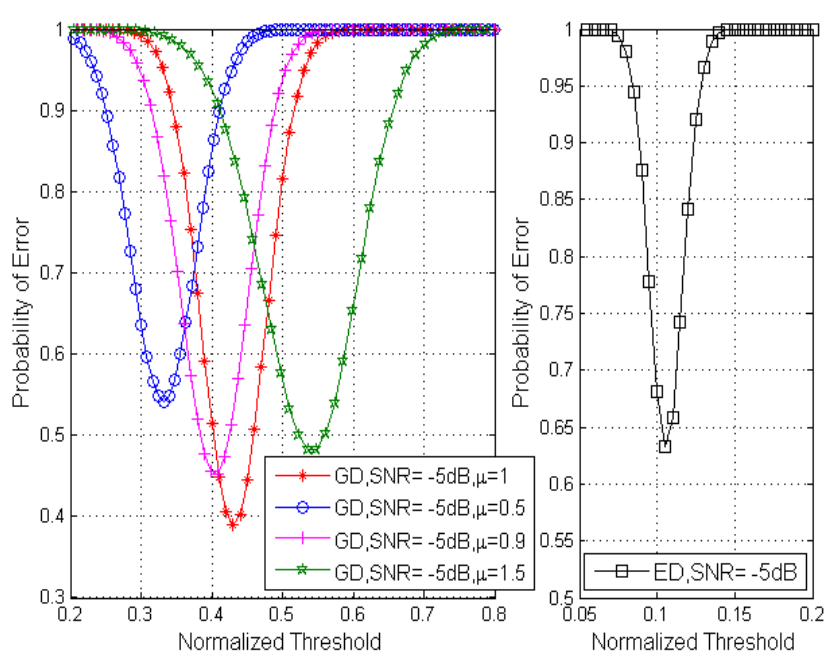

Fig. 10. The probability of error $P_{e r}$ for GD as a function of the normalized threshold at $\mu \neq 1\left(\sigma_{\xi}^{2} \neq \sigma_{\eta}^{2}\right)$. 
signals within the limits of GD AF bandwidth affecting the GD performance [26] and the problem of complete matching in all parameters between the model signal $s_{\bmod _{i}}[k]$ and the incoming PU signal $s_{i}[k]$ [33]. For example, the matching in amplitudes is possible only in the ideal case.

\section{Conclusions}

In this paper, the GD implementation in CR network as a spectrum sensor is investigated. The GD demonstrates the better spectrum sensing performance in comparison with the ED, WED, GLRT detector, and MME detector under the spatially correlated and independent or uncorrelated antenna array elements. In general, both for the GD and ED, the probability of false alarm $P_{F A}$ are low bounded if the antenna array elements are independent or uncorrelated. The GD performance character as a function of the coefficient of correlation between the antenna array elements is similar with the ED one while the use of the large number of antenna array elements allows us to reduce a negative effect of spatial correlation. The GD achieves the lower probability of error in comparison with the ED at the same $S N R$. The GD spectrum sensing performance is estimated in the case of equal variances of the noise at the PF and AF outputs, i.e. $\sigma_{\xi}^{2}=\sigma_{\eta}{ }^{2}(\mu=1$, the ideal case) and in the case of unequal variances, $\sigma_{\xi}^{2} \neq \sigma_{\eta}^{2}(\mu \neq 1$, the case in practice). As a result, we observe a superiority of GD implementation in CR network in comparison with the ED for both cases. Thus, we can conclude that even if we believe that the noise power at the GD PF and GD AF outputs are differed, the GD can still maintain the better spectrum sensing performance in comparison with ED.

\section{Appendix}

In the case of independent or uncorrelated antenna array elements, taking into consideration (59)-(62), (70), and (71), the GD optimal threshold $T H R_{G D}^{o p}$ can be derived using the factor of proportionality $\mu$ if the noise power at the $\mathrm{PF}$ and AF outputs is not the same in the following form:

$$
\begin{aligned}
T H R_{G D}^{o p} & =\underset{\sigma_{\xi}^{2} \neq \sigma_{\eta}^{2}}{\arg \min } \frac{1}{2} \\
& \times\left\{1-\frac{1}{2} \operatorname{erfc}\left\{\frac{T H R_{G D}-N M E_{s} \sigma_{h}^{2}}{\sqrt{2 N M\left[E_{s}^{2} \sigma_{h}^{4}+2 \sigma_{w}^{4}\left(1+\mu^{2}\right)\right]}}\right\}\right. \\
& \left.+\frac{1}{2} \operatorname{erfc}\left\{\frac{T H R_{G D}}{2 \sigma_{w}^{2} \sqrt{N M\left(1+\mu^{2}\right)}}\right\}\right\}
\end{aligned}
$$

We obtain (81) by the aid of

$$
Q(x)=\frac{1}{2} \operatorname{erfc}\left(\frac{x}{\sqrt{2}}\right)
$$

where

$$
\operatorname{erfc}(x)=\frac{2}{\sqrt{\pi}} \int_{x}^{\infty} e^{-t^{2}} d t
$$

is the complementary error function. We can determine the GD optimal threshold $T H R_{G D_{\sigma_{\xi}^{2} \neq \sigma_{\eta}^{2}}^{o p}}$ satisfying the following condition

$$
\frac{\partial\left\{P_{e r}\left(T H R_{G D}\right)\right\}}{\partial\left(T H R_{G D}\right)}=0
$$

to define a minimum of (81). Using the following equation [43]

$$
\frac{\partial}{\partial x} \operatorname{erfc}\left(\frac{x-A}{B}\right)=-\frac{2}{B \sqrt{\pi}} \exp \left[-\frac{(x-A)^{2}}{B^{2}}\right]
$$

where $A$ and $B$ are the arbitrary constants, and taking into consideration (81) and the condition (84) we can write

$$
\begin{gathered}
\frac{\partial\left[P_{\text {error }}^{G D}\left(T H R_{G D}\right)\right]}{\partial\left(T H R_{G D}\right)}=\frac{1}{2}\left\{\frac{1}{\sqrt{2 \pi N M\left[E_{s}^{2} \sigma_{h}^{4}+2 \sigma_{w}^{4}\left(1+\mu^{2}\right)\right.}}\right. \\
\quad \times \exp \left\{-\frac{\left[T H R_{G D}-N M E_{s} \sigma_{h}^{2}\right]^{2}}{2 N M\left[E_{s}^{2} \sigma_{h}^{4}+2 \sigma_{w}^{4}\left(1+\mu^{2}\right)\right]}\right\} \\
\left.-\frac{1}{2 \sqrt{\pi N M \sigma_{w}^{4}\left(1+\mu^{2}\right)}} \exp \left\{-\frac{T H R_{G D}^{2}}{4 N M \sigma_{w}^{4}\left(1+\mu^{2}\right)}\right\}\right\}=0 .
\end{gathered}
$$

We can rewrite (86) in the following form:

$$
\begin{aligned}
& \frac{1}{2}\left\{\frac{1}{\sqrt{2 \pi N M\left[E_{s}^{2} \sigma_{h}^{4}+2 \sigma_{w}^{4}\left(1+\mu^{2}\right)\right]}}\right. \\
& \times \exp \left\{-\frac{\left[T H R_{G D}-N M E_{s} \sigma_{h}^{2}\right]^{2}}{2 N M\left[E_{s}^{2} \sigma_{h}^{4}+2 \sigma_{w}^{4}\left(1+\mu^{2}\right)\right]}\right\} \\
&\left.-\frac{1}{2 \sqrt{\pi N M \sigma_{w}^{4}\left(1+\mu^{2}\right)}} \exp \left\{-\frac{T H R_{G D}^{2}}{4 N M \sigma_{w}^{4}\left(1+\mu^{2}\right)}\right\}\right\}=0 \\
& \text { or } \quad \frac{1}{2 \sqrt{2 \pi N M\left[E_{s}^{2} \sigma_{h}^{4}+2 \sigma_{w}^{4}\left(1+\mu^{2}\right)\right]}} \\
& \times \exp \left\{-\frac{\left[T H R_{G D}-N M E_{s} \sigma_{h}^{2}\right]^{2}}{2 N M\left[E_{s}^{2} \sigma_{h}^{4}+2 \sigma_{w}^{4}\left(1+\mu^{2}\right)\right]}\right\} \\
&=\frac{1}{4 \sigma_{w}^{2}\left(1+\mu^{2}\right)} \exp \left\{-\frac{T H R_{G D}^{2}}{4 N M \sigma_{w}^{4}\left(1+\mu^{2}\right)}\right\} .
\end{aligned}
$$$$
\text { or }
$$

We can rewrite (88) as

$$
\frac{\exp \left\{-\frac{\left[T H R_{G D}-N M E_{s} \sigma_{h}^{2}\right]^{2}}{2 N M\left[E_{s}^{2} \sigma_{h}^{4}+2 \sigma_{w}^{4}\left(1+\mu^{2}\right)\right]}\right\}}{\exp \left\{-\frac{T H R_{G D}^{2}}{4 N M \sigma_{w}^{4}\left(1+\beta^{2}\right)}\right\}}
$$




$$
\begin{gathered}
=\sqrt{\frac{2\left[E_{s}^{2} \sigma_{h}^{4}+2 \sigma_{w}^{4}\left(1+\mu^{2}\right)\right]}{4 \sigma_{w}^{4}\left(1+\mu^{2}\right)^{2}}} \\
\operatorname{or} \exp \left\{-\frac{\left[T H R_{G D}-N M E_{s} \sigma_{h}^{2}\right]^{2}}{2 N M\left[E_{s}^{2} \sigma_{h}^{4}+2 \sigma_{w}^{4}\left(1+\mu^{2}\right)\right]}+\frac{T H R_{G D}^{2}}{4 N M \sigma_{w}^{4}\left(1+\mu^{2}\right)}\right\} \\
=\sqrt{\frac{2\left[E_{s}^{2} \sigma_{h}^{4}+2 \sigma_{w}^{4}\left(1+\mu^{2}\right)\right]}{4 \sigma_{w}^{4}\left(1+\mu^{2}\right)^{2}}}
\end{gathered}
$$

Taking the natural logarithm, we obtain

$$
\begin{gathered}
-\frac{\left[T H R_{G D}-N M E_{s} \sigma_{h}^{2}\right]^{2}}{2 N M\left[E_{s}^{2} \sigma_{h}^{4}+2 \sigma_{w}^{4}\left(1+\mu^{2}\right)\right]}+\frac{T H R_{G D}^{2}}{4 N M \sigma_{w}^{4}\left(1+\mu^{2}\right)} \\
=\ln \sqrt{\frac{2\left[E_{s}^{2} \sigma_{h}^{4}+2 \sigma_{w}^{4}\left(1+\mu^{2}\right)\right]}{4 \sigma_{w}^{4}\left(1+\mu^{2}\right)^{2}}} .
\end{gathered}
$$

Taking into consideration that $S N R$ in cognitive radio networks is very small, i.e. $\sigma_{w}^{2} \gg>1$ we can think that $\frac{\sqrt{E_{s}^{2} \sigma_{h}^{4}+4 \sigma_{w}^{4}}}{2 \sigma_{w}^{2}} \rightarrow 1$ and $\ln \frac{\sqrt{E_{s}^{2} \sigma_{h}^{4}+4 \sigma_{w}^{4}}}{2 \sigma_{w}^{2}} \rightarrow 0$. After some mathematical transformations we obtain

$$
\begin{gathered}
E_{s}^{2} \sigma_{h}^{4} \times T H R_{G D}^{2}+4 N M E_{s} \sigma_{h}^{2} \sigma_{w}^{4}\left(1+\mu^{2}\right) \times T H R_{G D} \\
-2 N^{2} M^{2} E_{s}^{2} \sigma_{h}^{4} \sigma_{w}^{4}\left(1+\mu^{2}\right)=0 .
\end{gathered}
$$

Solution of the quadratic equation (92) is well-known

$$
\begin{gathered}
T H R_{G D}^{o p}=\frac{-b \pm \sqrt{b^{2}-4 a c}}{2 a}, \\
\left\{\begin{array}{l}
a=E_{s}^{2} \sigma_{h}^{4} ; \\
b=4 N M E_{s} \sigma_{h}^{2} \sigma_{w}^{4}\left(1+\mu^{2}\right) ; \\
c=-4 N^{2} M^{2} E_{s}^{2} \sigma_{h}^{4} \sigma_{w}^{4}\left(1+\mu^{2}\right) .
\end{array}\right.
\end{gathered}
$$

Substituting (94) into (93) and taking into consideration that the GD threshold is defined by the absolute value as it is discussed in [18, Chapter 7, p. 679-685], we obtain

$$
T H R_{G D_{\sigma_{\xi}^{2} \neq \sigma_{\eta}^{2}}^{o p}}=N M \sigma_{w}^{2} \sqrt{2\left(1+\mu^{2}\right)} .
$$

As follows from (95), if $\mu=1$ we obtain the optimal GD detection threshold in the following form

$$
T H R_{G D_{\sigma_{\xi}^{2}=\sigma_{\eta}^{2}}^{o p}}=2 N M \sigma_{w}^{2} .
$$

\section{Acknowledgements}

This research was supported by the Kyungpook National University Research Fund 2014. Additionally, the authors would like to thank the anonymous reviewers for the comments and suggestions that helped to improve a quality of the present paper.

\section{References}

[1] YUCEK, T., ARSLAN, H. A survey of spectrum sensing algorithms for cognitive radio applications. IEEE Communications Surveys and Tutorials, 2009, vol. 11, no. 1, p. 116-130. DOI: 10.1109/SURV.2009.090109

[2] SONNENSCHEIN, A., FISHMAN, P.M. Radiometric detection of spread spectrum signals in noise of uncertainty power. IEEE Transactions on Aerospace and Electronic Systems, 1992, vol. 28, no. 3, p. 654-660. DOI: 10.1109/7.256287

[3] KAY, S. M. Fundamentals of Statistical Signal Processing: Detection Theory. Vol. 2. New Jersey, USA: Prentice Hall, 1998.

[4] CHEN, H.S., GAO, W., DAUT, D. G. Signature based spectrum sensing algorithms for IEEE 802.22 WRAN. In Proceedings of the IEEE International Conf. on Communications (ICC'07). Glasgow (Scotland), 2007, p. 6487-6492. DOI: 10.1109/ICC.2007.1073

[5] CARBIC, D., TKACHENKO, A., BRODERSEN, R. W. Spectrum sensing measurements of pilot, energy, and collaborative detection. In Proc. of the IEEE Military Communications Conf. Washington (USA), 2006, 7 p. DOI: 10.1109/MILCOM.2006.301994

[6] GARDNER, W. A. Exploitation of spectral redundancy in cyclostationary signals. IEEE Signal Processing Magazine, 1991, no. 8, p. 14-36. DOI: 10.1109/79.81007

[7] HAN, N., SHON, S. H., JOO, J. O., KIM, J. M. Spectra correlation based signal detection method for spectrum sensing in IEEE 802. 22 WRAN systems. In Proceedings of the $8^{\text {th }}$ International Conference on Advanced Communications Technology. Gangwon-Do (South Korea), 2006, p. 1765-1770.

[8] YU, G., LONG, C., XIANG, M., XI, W. A. Novel energy detection scheme based on dynamic threshold in cognitive radio systems. Journal of Computational Information Systems, 2012, vol. 8, no. 6 , p. 2245-2252.

[9] CHEN, H., LIU, J. Cooperative spectrum sensing based on double threshold detection and Dempster-Shafer theory. In Proceedings of the $12^{\text {th }}$ IEEE International Conference on Communications Technology (ICCT'2010). Nanjing (China), 2010, p. 1212-1215. DOI: $10.1109 /$ ICCT.2010.5689114

[10] LUO, L. P., ZHANG, P., ZHANG, G. C., QIN, J. Y. Spectrum sensing for cognitive radio networks with correlated multiple antennas. Electronics Letters, 2011, vol. 47, no. 23, p. 1297-1298. DOI: $10.1049 / \mathrm{el} .2011 .2231$

[11] XIAO, X., LIU, S., LU, K., WANG, J. MaxDiv: an optimal randomized spectrum access with maximum diversity scheme for cognitive radio networks. International Journal on Communication Systems, 2012, vol. 25, p.832-848. DOI: 10.1002/dac.1291.

[12] QUAN, Z., CUI, S., SAYED, A. H. Optimal linear cooperative for spectrum sensing in cognitive radio networks. IEEE Journal of Selected Topics in Signal Processing, 2008, vol. 2, no. 1, p. 28-40. DOI: 10.1109/JSTSP.2007.914882

[13] LIM, T. J., ZGANG, R., LIANG, Y. C., ZENG, Y. GLRT-based spectrum sensing for cognitive radio. In Proc. of the IEEE Global Telecommunications Conf. (GLOBECOM'08). New Orleans (USA), 2008, p. 1-5. DOI: 10.1109/GLOCOM.2008.ECP.842

[14] ZENG, Y., LIANG, Y. C. Maximum-minimum eigenvalue detection for cognitive radio. In Proc. of the IEEE $18^{\text {th }}$ Internat. Symp. on Personal, Indoor and Mobile Radio Communications (PIMRC'07). Athens (Greece), 2007, p. 1-5.

[15] MEHTA, M., DREW, N., VARDOUliAS, G., GRECO, N., NIEDERMEIER, C. Reconfigurable terminals: an overview of architectural solutions. IEEE Communications Magazine, 2001, vol. 39, no. 8, p. 82-89. DOI: 10.1109/35.940041

[16] GHAVAMI, S., ABOLHASSANI, B. Spectrum sensing and power 
/rate control in CDMA cognitive radio networks. International Journal on Communications Systems, 2011, vol. 24, p.121-145. DOI: $10.1002 /$ dac. 1258

[17] TUZLUKOV, V. A new approach to signal detection theory. Digital Signal Processing: A Review Journal, 1998, vol. 8, no. 3 , p. $166-184$. DOI: $10.1006 /$ dspr.1998.0315

[18] TUZLUKOV, V. Signal Detection Theory. Boston (USA): Springer-Verlag, 2001.

[19] TUZLUKOV, V. Generalized approach to signal processing in wireless communications: the main aspects and some examples. Chapter 11. Wireless Communications and Networks - Recent Advances. Rijeka, Croatia: InTech, 2012, p. 305-338.

[20] TUZLUKOV, V. DS-CDMA downlink systems with fading channel employing the generalized receiver. Digital Signal Processing, 2011, vol. 21, no. 6, p. 725-733. DOI: 10.1016/j.dsp.2011.07.005

[21] TUZLUKOV, V. Signal processing by generalized receiver in DSCDMA wireless communication systems with frequency-selective channels. Circuits, Systems Signal Processing, 2011, vol. 30, no. 6, p. 1197-1230. DOI: $10.1007 / \mathrm{s} 00034-011-9273-1$

[22] Communication Systems: New Research. Editor: Tuzlukov, V. New York (USA): NOVA Science Publishers, Inc., 2013, 423 p.

[23] TUZLUKOV, V. Wireless communications: Generalized approach to signal processing. Chapter 6. Communication Systems: New Research. Editor: Tuzlukov, V. New York (USA): NOVA Science Publishers, Inc., 2013, p.175-268.

[24] TUZLUKOV, V. Signal processing by generalized receiver in DSCDMA wireless communication systems. Chapter 4. Contemporary Issues in Wireless Communications. Rijeka, Croatia: InTech, 2014, p. 79-158.

[25] TUZLUKOV, V. Signal Processing in Radar Systems. Taylor \& Francis Group: CRC Press, 2012.

[26] SHBAT, M., TUZLUKOV, V. Evaluation of detection performance under employment of the generalized detector in radar sensor systems. Radioengineering, 2014 , vol. 23 , no. 1, p. 50-65.

[27] SHBAT, M., TUZLUKOV, V. Definition of adaptive detection threshold under employment of the generalized detector in radar sensor systems. IET Signal Processing, 2014, vol. 8, no. 6, p. 622-632. DOI: 10.1049/iet-spr.2013.0235

[28] SHBAT, M., TUZLUKOV, V. Spectrum sensing under correlated antenna array using generalized detector in cognitive radio systems. International Journal of Antennas and Propagation. 2013. Article ID 853746,8 pages, DOI: $10.1155 / 2013 / 853746$

[29] KIM, S., LEE, J., WANG, H., HONG, D. Sensing performance of energy detector with correlated multiple antennas. IEEE Signal Processing Letters, 2009, vol. 16, no. 8, p. 671-674. DOI: 10.1109/LSP.2009.2021381

[30] JIN, M., LI, Y., ZHANG, R., WANG, R. A new spectrum sensing algorithm based on antenna correlation for cognitive radio systems. Wireless Personal Communications, 2012, vol. 66, p. 419-428, DOI: $10.1007 / \mathrm{s} 11277-011-0349-9$

[31] MOLISCH, A., GREENSTEIN, L., SHAFI, M. Propagation issues for cognitive radio. IEEE Proceedings. 2009, vol. 97, no. 5, p. 787-804. DOI: 0.1109/JPROC.2009.2015704

[32] LOYKA, S. Channel capacity of MIMO architecture using the exponential correlation matrix. IEEE Communications Letters, 2001, no. 5, p. 369-371. DOI: $10.1109 / 4234.951380$

[33] SHBAT, M. S. Performance analysis of signal detection by GD in radar sensor and cognitive radio systems. PhD Thesis. Kyungpook National University, February 2014.

[34] MAXIMOV, M. Joint correlation of fluctuative noise at outputs of frequency filters. Radio Engineering, 1956, no. 9, p. 28-38.
[35] CHERNYAK, Yu. Joint correlation of noise voltage at outputs of amplifiers with nonoverlapping responses. Radio Physics and Electronics, 1960, no. 4, p. 551-561.

[36] TUZLUKOV, V. Signal Processing Noise. Boca Raton, London, New York, Washington D.C.: CRC Press, Taylor \& Francis Group, 2002.

[37] TABOGA, M. Lectures on Probability Theory and Mathematical Statistics. $2^{\text {nd }}$ Ed. Amazon Create Space, 2012.

[38] GRADSHTEYN, D.C., RYZHIK, I. Table of Integrals, Series, and Products. $7^{\text {th }}$ ed. Academic Press: 2007.

[39] ALOUINI, M. S., ABDI, A., KAVEH, M. Sum of gamma variates and performance of wireless communications systems over Nakagami-fading channels. IEEE Transactions on Vehicular Technology, 2001, vol. 50, no. 6, p. 1471-1480. DOI: $10.1109 / 25.966578$

[40] LIANG, Y. C., ZENG, Y., PEH, E, HOANG, A. T. Sensingthroughput trade off for cognitive radio networks. IEEE Transactions on Wireless Communications, 2008, vol. 7, no. 4, p. 1326-1337. DOI: 0.1109/TWC.2008.060869

[41] ZHANG, X., WU, Q., WANG, J. Optimization of sensing time in multichannel sequential sensing for cognitive radio. International Journal on Communication Systems, 2013, vol. 26, p. 222-235. DOI: $10.1002 /$ dac. 1341

[42] ATAPATtU, S., TellambuRA, C., JiANG, H. Spectrum sensing via energy detector in low SNR. In Proc. of the IEEE International Conf. on Communications (ICC'11). Kyoto (Japan), 2011, p. 1-5. DOI: 10.1109/icc.2011.5963316

[43] KATE, S. K., NAVARE, S. D., BANDEWAR, S. R., et al. Engineering Mathematics-II. India: Technical Publications Pune, 2007.

\section{About the Authors ...}

Modar Safir SHBAT was born in 1980. He received his B.E degree in Electronic Engineering from Damascus University in 2003, M.S. degree in Information Technology and Science from the Korean Advanced Inst. of Science and Technology (KAIST), Dajeon, South Korea in 2008 and Ph.D. degree from the College of IT Engineering, Kyungpook National University, Daegu, South Korea in 2014. His research interests include radio resource management, wireless mobile networks, cognitive radio networks, radar signal processing.

Vyacheslav TUZLUKOV was born in 1954. He received his M.S. degree in Radiophysics and Electronics and Ph.D. degree in Radiophysics from the Belarusian State University, Minsk, Belarus in 1976 and 1990, respectively. At the present time, Dr. Tuzlukov is a Director of Signal Processing Laboratory and Full Professor at the Dept. of Information Technologies and Communication Engineering, School of Electronics Engineering, College of IT Engineering, Kyungpook National University, Daegu, South Korea. $\mathrm{He}$ is an author over 200 journal and conference papers and ten monographs in signal processing and has also contributed the Chapters in nine books on signal processing in wireless communications and radar. His research emphasis is on signal processing in wireless communications, wireless sensor networks, cognitive radio networks, radar/sonar, remote sensing, mobile communications, etc. 